\title{
EXPERIMENTAL COMPRESSION TESTS OF COLD- FORMED STEEL (CFS) TO VERIFY ITS CODE-BASED STRENGTH
}

\author{
Bernardo Lejano ${ }^{1}$, James Matthew De Jesus ${ }^{2}$, and Arvin Patrick $\mathbf{Y u}^{3}$ \\ ${ }^{1}$ Civil Engineering Department, Gokongwei College of Engineering, De La Salle University, Manila, Philippines, \\ Tel: +6325244611 local 226, e-mail: bernardo.lejano@dlsu.edu.ph \\ 2DCCD Engineering Corporation, Makati, Philippines, e-mail: james_dejesus@dlsu.edu.ph \\ ${ }^{3}$ Monolith Construction and Development Corporation, Quezon City, Philippines
}

Received Date: July 19, 2018; Revised Date: February 25, 2020; Acceptance Date: August 10, 2020

\begin{abstract}
Cold-Formed Steel (CFS) is a good construction material because of its high strength-to-weight ratio, that is, it exhibits efficient load carrying capabilities in combination with its lightweight characteristics. Although CFS is already being used in construction, information on structural performance of locallyproduced CFS in the Philippines is scarce. To date, the authors have not found any experimental study done in the Philippines regarding the structural performance of locally-produced CFS. In this study, C-section and Z-section are being studied since these members exhibit buckling failures that may be difficult to predict due to complexity of their section geometry. The objective of this paper is to present the performance of these CFS sections when subjected to concentric axial compression both experimentally and computationally. For the experimental part, the CFS members were subjected to axial compression using a hydraulic jack. High-speed video cameras were used to capture the different failure modes. For the computational aspect, provisions found in the National Structural Code of the Philippines (NSCP) were used to calculate the compression strength of the members. A total of $80 \mathrm{C}$-section specimens with 5 different lengths and 5 different thicknesses were tested. It was found that the strength calculations using the NSCP provisions were not consistent with the results of the compression tests. For shorter lengths, distortional buckling prevailed as the main failure, while for longer lengths, torsional-flexural buckling occurred. All of the predicted strengths were highly conservative. For the Z-section, a total of 180 specimens with 6 different lengths and 6 different thicknesses were tested. Torsional-flexural buckling was observed in majority of the specimens. Although most of the failure modes were predicted correctly, it was found that the predicted strengths using the NSCP were relatively high compared to the experimental results, thus non-conservative. Finite Element Method (FEM) analyses using ANSYS were conducted. Findings indicate that the experiment results agreed well with the FEM results.
\end{abstract}

Keywords: Axial compression, Buckling, C-section, Cold-formed steel, Z-section

\section{Introduction}

There is very limited technical information about Cold-Formed Steel (CFS) in the Philippines and yet it is used in construction of structures. Specifically, there is a gap in experimental studies done for locally produced CFS. In recent years, application of cold-formed steel for structural purpose has increased here and abroad. Davies has reviewed the advancement of CFS in terms of material 
quality, section properties, interaction of failures, and the widening scope of application [1]. Mostly, it is used as residential and low-rise buildings construction in the United States. Rondal backed up this claim and further stated that cold-formed steel members have reached the "age of maturity" [2]. However, the use of thinner sections and higher yield strengths can lead to structural design problems according to Dubina et al., and Hancock [3, 4]. These are due to its complexity that is not routinely encountered by most structural engineers. To allow for safety, the structure must be meticulously analyzed in accordance with code provisions or other methods such as performance-based analysis.

Currently, there are design provisions for CFS members in Chapter 5 of the National Structural Code of the Philippines (hereafter will just be referred to as "NSCP") that can be used by structural engineers. The provisions provide formulas for the computation of the compressive strength of C and Z-sections CFS [5]. However, these provisions in the NSCP were based on design standards formulated in other countries. Although the steel used may be the same, the process of local manufacturing may have slight variation that might affect its performance.

Over the years, the NSCP has been regarded as the basis of design of structures all over the Philippines. The provisions in the NSCP are assumed to be correct and safe. Confidence in safety is achieved when the provisions in the NSCP are religiously followed. However, the design provisions of $\mathrm{C}$ and Z-sections CFS have not been fully verified in the field. Experimental tests are needed to confirm the accuracy of their design. According to Rondal, CFS can be unstable in different failure modes such as local, distortional and flexural or torsional-flexural buckling when subjected to axial compression [2].

The main objective of this study is to investigate the load carrying-capacity of $\mathrm{C}$ and $\mathrm{Z}$ CFS subjected to axial compression. The specific objectives are to investigate the different buckling modes and to evaluate the axial strength based on the NSCP and experimental tests.

\section{Methodology}

This study focused on experimental and computational methods of research. The experimental method of this study is to conduct compressive tests on $\mathrm{C}$ and Z-sections cold-formed steel to acquire its strength and mode of failure. The computational aspect of the study is done by using the formulas recommended by the NSCP in determining the strength and mode of failure of each member. The critical loads obtained from experiment and calculations are compared to verify the reliability of the NSCP provisions and if needed verify this using Finite Element Method (FEM).

\section{Specimens}

The shape of the section investigated is in the form of a $\mathrm{C}$ and $\mathrm{Z}$-section. For reference, the typical cross-sections of $\mathrm{C}$ and $\mathrm{Z}$-section CFS are shown in Figure 1.

Based on demand and availability in the market, the CFSs used in this study are LC90x35x5 for C-section and LZ100x50x20 for Z-section. Both are with simple lip stiffeners as stipulated in section 553.4 of the NSCP. For the C-section, specimens were tested with variation of 5 thicknesses and 5 lengths. A total of 80 specimens were tested for this batch, 4 specimens for the 20 cases considered. For the Z-section, six thicknesses and six lengths were considered 
resulting to 36 cases, with five test specimens per case, for a total of 180 test specimens. Shown in Table 1 is the label or code used for the specimens. Those starting with $\mathrm{C}$ refer to the $\mathrm{C}$-section CFS specimens, and those starting with Z refer to the Z-section CFS specimens. For example, C1A refer to a specimen with C-section having a nominal length of $800 \mathrm{~mm}$ and nominal thickness of $0.40 \mathrm{~mm}$ (refer to Table 1). The average section properties for each section shape are also tabulated in Table 2.

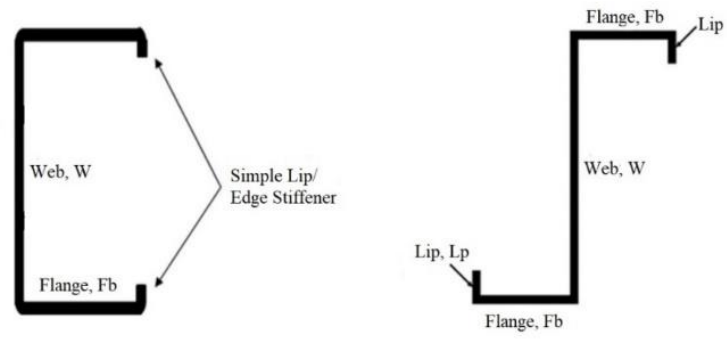

Figure 1. Cross-section of c-section and z-section cold-formed steel

Table 1. Code Used for the Specimen

Length Code

\section{Thickness Code}

Nominal Thickness (mm)

\begin{tabular}{lccc}
\hline C1 & 800 & A & 0.40 \\
C2 & 1100 & B & 0.52 \\
C3 & 1400 & $\mathrm{C}$ & 0.60 \\
C4 & 1800 & $\mathrm{D}$ & 0.80 \\
C5 & 2000 & $\mathrm{E}$ & 1.00 \\
Z1 & 800 & $\mathrm{~A}$ & 0.80 \\
$\mathrm{Z} 2$ & 1100 & $\mathrm{~B}$ & 1.00 \\
$\mathrm{Z} 3$ & 1400 & $\mathrm{C}$ & 1.20 \\
$\mathrm{Z} 4$ & 1700 & $\mathrm{D}$ & 1.40 \\
$\mathrm{Z} 5$ & 1800 & $\mathrm{E}$ & 1.50 \\
$\mathrm{Z} 6$ & 2000 & $\mathrm{~F}$ & 1.80 \\
\hline
\end{tabular}

Table 2. Average Section Properties

\begin{tabular}{cccccc} 
Section & Web $(\mathbf{m m})$ & $\begin{array}{c}\text { Top Flange } \\
(\mathbf{m m})\end{array}$ & $\begin{array}{c}\text { Bottom } \\
\text { Flange }(\mathbf{m m})\end{array}$ & $\begin{array}{c}\text { Top Lip } \\
(\mathbf{m m})\end{array}$ & $\begin{array}{c}\text { Bottom Lip } \\
(\mathbf{m m})\end{array}$ \\
\hline C & 87.97 & 38.15 & 38.26 & 6.86 & 6.86 \\
Z & 101.56 & 50.13 & 50.02 & 18.76 & 19.03 \\
\hline
\end{tabular}

To ensure that calculations were accurate, actual measurements of the dimensions of the specimens were taken prior to test of each specimen. The measurements were taken using a digital caliper measuring two flanges, two lips, web, thickness and overall height. Each element of the cross-section together with the thickness and length were measured three times at approximately three equidistant sections (e.g. top, middle \& bottom). 
Material properties that were considered to affect the strength and failure modes of the member are the yield strength, Fy, and modulus of elasticity, E. These material properties were determined using ASTM E8: Standard Test Methods for Tension Testing of Metallic Materials where strips of metal were cut and tested from the specimen population of CFS [6]. These material properties together with the dimensional parameters were used in determining the effective width of the web, flange and lip elements to finally determine the nominal strength.

\section{Experimental Test setup}

The member specimens were loaded axially in compression with the use of a hydraulic jack. The compression load was gradually applied to each specimen. Load cell was placed right below the hydraulic jack to monitor the load that was applied. To simplify the calculation of the axial force, the end conditions were designed to be pin-supported, that is, the supports are free to twist and bend. To simulate the pin-ended condition of the member, steel end caps were placed in both ends of the member with a $20 \mathrm{~mm}$ diameter bearing ball attached to provide a ball and socket mechanism for the specimen to rotate freely about its ends. The ball was positioned so that the line of action of the force will pass through the centroid of the cross-section of the specimen so that the members can be said to be concentrically-loaded [7,8]. The experimental set-up is shown in Figure 2. It shows how the specimens are set-up in the standing steel frame. Also shown in the figure are the picture of the top view and front view of the test set-up.

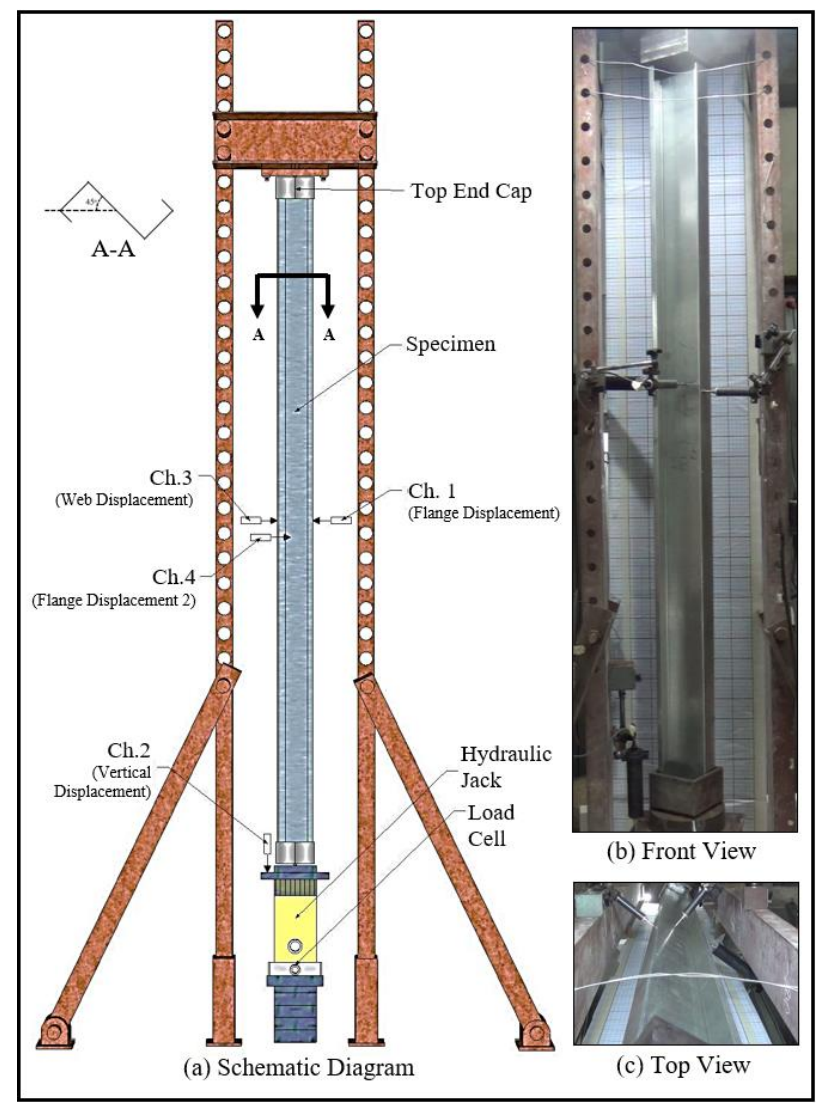

Figure 2. Experimental test setup 
Two displacement transducers were placed in both flanges to record the distortional deformation. Another transducer was also placed in the web to record flexural and/or torsional movement. These three transducers were placed approximately at the midspan. Another transducer was placed at the bottom cap to measure the vertical displacement. Load cells and transducers were used to measure the experimental data for strength, displacement and deformation to provide accurate results. All these measuring devices were connected to a data logger. The applied load, element movements and longitudinal movement were recorded in the data logger which was able to record all four displacements and the load applied to the member. High-speed cameras were also used to observe the governing failure mode, which is the buckling deformation at failure.

\section{Failure Modes}

Three failure modes were considered for the CFS members in concentric compression. They are local buckling, torsional or flexural-torsional buckling and distortional buckling. Full yielding, which is the failure when of the stress in whole cross section reach yield stress, is not considered anymore because of the thinness of the member which is more prone to buckling. Local buckling happens when the failure is caused by buckling of an element in a particular local portion of the member. Torsional or flexural torsional buckling is due to the lateral displacement that occurs, creating twisting and bending of the member. Sketch of these failure modes are shown in Figure 3. Distortional failure happens when the flanges rotate about the web junction displacing the flange from its original position. In addition, the effectiveness of the elements of the section was taken into account. With the effect of local buckling on the section, the width of each element was reduced thus resulting to a lesser cross-sectional area than the full area. Formulas found in NSCP Sections 552 and 553 were used [5].

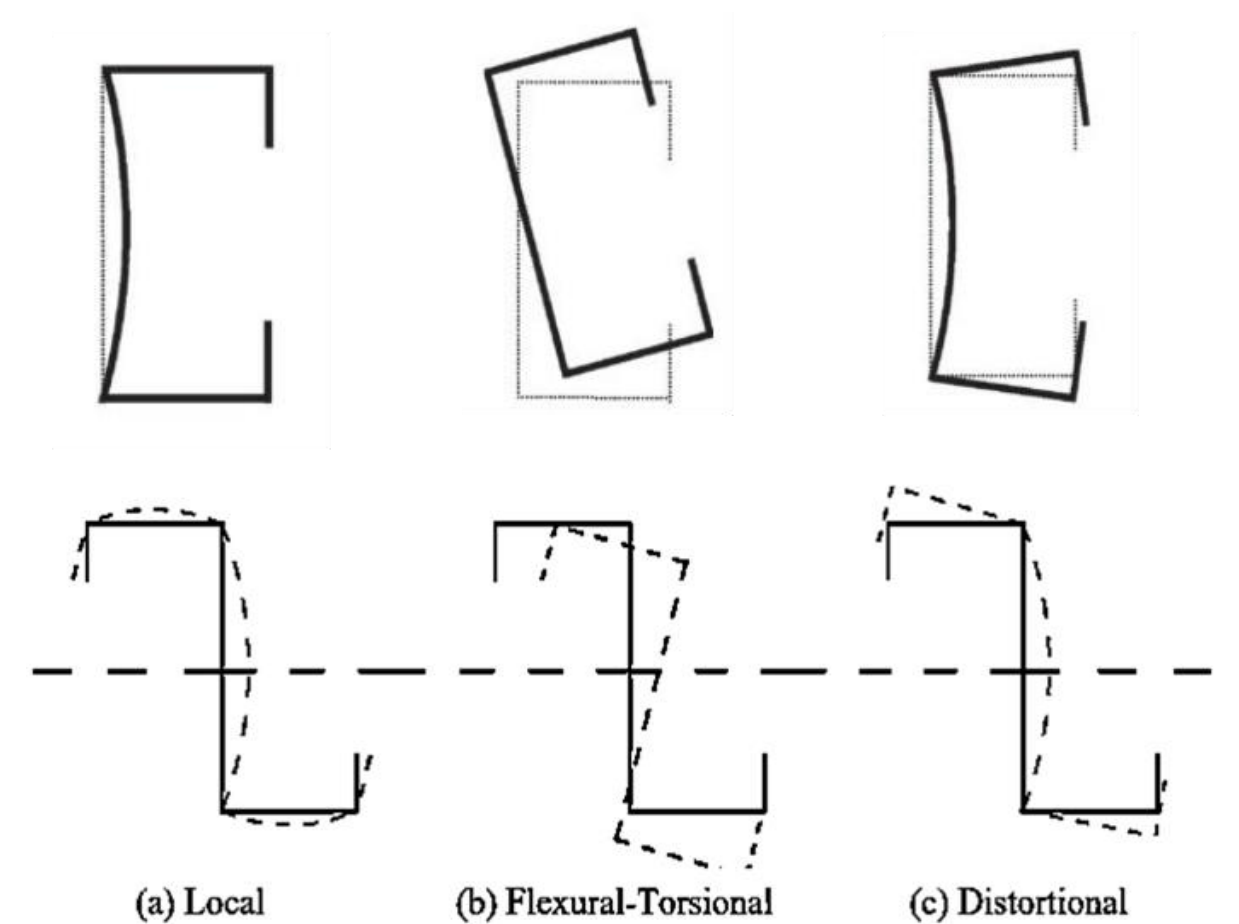

Figure 3. Section buckling modes of failure 
Buckling was monitored and identified using the high-speed cameras. The displacement transducers were also used as basis to determine the movements of the member as it is loaded in compression. Local buckling was usually encountered first. Even if local buckling transpired, it was further loaded to see the buckling mode that the member will exhibit. The different modes of failure are illustrated in Figure 3. However, the figures just demonstrate the general movements of the elements for each mode. The movement of the elements can either be inward or outward which makes the failure modes more complex. Consequently, a forward or backward movement can also transpire. Furthermore, there exists a local-global interaction in buckling failure modes such that multiple modes can be exhibited by a member specimen as reported by Batista [9].

\section{Results and Discussion}

The calculation of strength depends heavily on the values of the yield strength and modulus of elasticity of Galvanized Iron sheets or G.I. sheets from where the CFS was made from. For the Csection, test results indicate an average yield strength obtained was $242.45 \mathrm{MPa}$ which is a typical strength for galvanized iron sheets. Furthermore, the average modulus of elasticity was $28.05 \mathrm{GPa}$. For the Z-section, average yield strength obtained was 306.04 and the average modulus of elasticity from the test was $23.99 \mathrm{GPa}$.

\section{Evaluation of Strength through Experiment}

The compressive strengths of the CFS were evaluated from the compression tests of the C-section and Z-section specimens with varying thickness and length. These strengths will be termed as "experimental strengths", and denoted as Pexpt. The average values of the experimental strength results are summarized in matrix form in Table 3.

Table 3. Experimental Strength Test Results, Pexpt (kN)

\begin{tabular}{ccccccc} 
Code & C1 & C2 & C3 & C4 & C5 & \\
\hline A & 1.86 & 3.12 & 3.38 & $*$ & 1.23 & Legend: \\
B & 3.74 & 6.68 & 5.48 & 2.55 & 2.46 & $*$ Lost \\
C & 5.08 & 7.60 & 6.18 & 4.76 & 2.50 & data or \\
D & 6.86 & 10.42 & 9.10 & $*$ & 3.50 & corrupted \\
E & 10.06 & $*$ & $*$ & 9.18 & $*$ & data \\
\hline Code & $\mathbf{Z 1}$ & $\mathbf{Z 2}$ & $\mathbf{Z 3}$ & $\mathbf{Z 4}$ & $\mathbf{Z 5}$ & $\mathbf{Z 6}$ \\
\hline A & 1.64 & 1.37 & 0.93 & 1.19 & 1.20 & 0.79 \\
B & 2.41 & 2.06 & 1.94 & 1.87 & 1.39 & 1.46 \\
C & 3.21 & 2.92 & 2.73 & 2.51 & 2.45 & 2.13 \\
D & 4.96 & 4.33 & 4.17 & 3.31 & 2.84 & 2.77 \\
E & 5.08 & 4.13 & 4.80 & 4.06 & 3.36 & 3.09 \\
F & 8.16 & 6.93 & 5.84 & 5.18 & 4.65 & 3.73 \\
\hline
\end{tabular}

As seen in Table 3, the experimental strengths were affected by the thickness and the length. The thicker the member the higher the load it can carry. The longer the member, the lower is the strength. The length and thickness also influence the buckling mode of failure. Thickness governs the local buckling susceptibility of the individual elements. The length influences the global buckling susceptibility of the member. 
The observation of the failure modes was done by identifying the movement both locally and globally. Local, torsional-flexural and distortional buckling conditions were the main failure modes to be identified. High speed camera captured pictures at the onset of failure were used as basis in determining the failure mode. The failure modes observed were torsional-flexural buckling (TF) and distortional buckling (DB). As example, Figure 4 shows observed torsional-flexural (TF) and distortional buckling (DB).
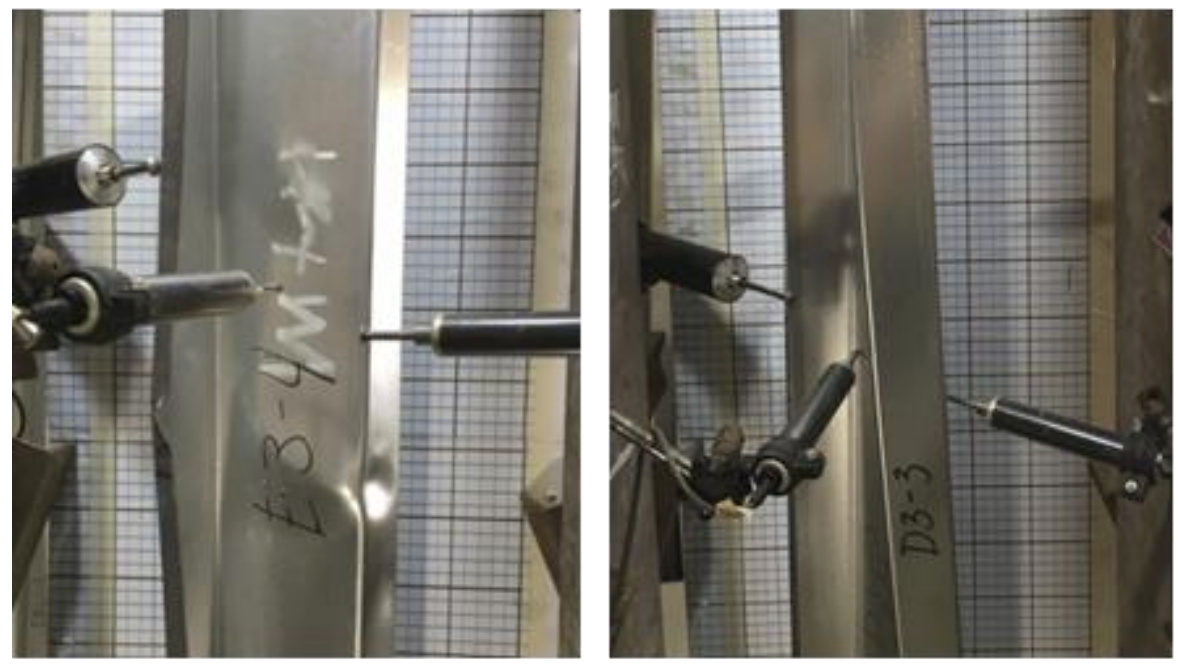

Figure 4. Failure due to torsional-flexural bucking (right) and distortional buckling (left)

\section{Evaluation of Strength through Computation}

The computations of the strength of the members, termed as "computational strength" and denoted as Pcalc, were done using the formulas and provisions stipulated in the NSCP for the design strength of Cold-Formed Steel compression members. These provisions are summarized in NSCP Sections 552 and 553. The NSCP considers three main failure modes. These are yielding, torsionalflexural and distortional buckling. The computational strength considered were based on the provisions of the NSCP was taken as the lowest load amongst the three failure modes [5]. Although the yielding strength is generally unattainable, it was still computed for comparison with the buckling strength. The computational strength results are summarized in Table 4. The same influence of thickness and length is observed in the computational strength, that is: the longer the length, the lower the strength; and the thicker the member, the higher the strength. As seen in Table 4 , the combination of shorter length and a thicker GI sheet resulted to the larger computational strength.

To evaluate the prediction of the NSCP formulas with the experiment results, the ratio of Pext against Pcalc were computed. This will be referred to as the strength ratio. The results are tabulated in Table 5. It can be seen that the values are greater than 1 for the $\mathrm{C}$-section. This means that the predictions using the NSCP formulas for the C-sections are very conservative. However, for the Z-section CFS, the ratio Pexpt/Pcalc is lower than 1 . This means that it would be an overestimation to use the NSCP formulas for Z-section. Moreover, the average ratio for the $\mathrm{C}$-section is 2.56 while the average strength ratio for Z-section is 0.50 . 
Table 4. Computational Strength Results, (Pcalc, kN)

\begin{tabular}{ccccccc} 
Code & C1 & C2 & C3 & C4 & C5 & \\
\hline A & 0.89 & 1.02 & 1.04 & $*$ & 0.88 & Legend: \\
B & 1.62 & 1.41 & 2.17 & 1.60 & 1.38 & * Lost \\
C & 1.77 & 1.84 & 2.48 & 2.59 & 1.72 & data or \\
D & 2.03 & 4.17 & 3.45 & $*$ & 2.23 & corrupted \\
E & 3.25 & $*$ & $*$ & 3.75 & $*$ & data \\
\hline Code & $\mathbf{Z 1}$ & $\mathbf{Z 2}$ & $\mathbf{Z 3}$ & $\mathbf{Z 4}$ & $\mathbf{Z 5}$ & $\mathbf{Z 6}$ \\
\hline A & 4.73 & 4.88 & 4.03 & 3.29 & 3.13 & 2.77 \\
B & 7.42 & 6.53 & 4.79 & 4.01 & 3.90 & 3.46 \\
C & 8.67 & 6.97 & 5.57 & 4.74 & 4.42 & 3.93 \\
D & 11.73 & 8.57 & 6.55 & 5.33 & 4.91 & 4.42 \\
E & 12.50 & 9.25 & 7.04 & 5.72 & 5.15 & 4.69 \\
F & 14.71 & 10.90 & 7.97 & 6.53 & 6.20 & 5.70 \\
\hline
\end{tabular}

Table 5. Ratio of Experimental Against Calculated Strength (Pexpt/Pcalc)

\begin{tabular}{ccccccc} 
Code & C1 & C2 & C3 & C4 & C5 & \\
\hline A & 2.09 & 3.06 & 3.25 & $*$ & 1.40 & Legend: \\
B & 2.31 & 4.74 & 2.53 & 1.59 & 1.78 & * Lost \\
C & 2.87 & 4.13 & 2.49 & 1.84 & 1.45 & data or \\
D & 3.38 & 2.50 & 2.64 & $*$ & 1.57 & corrupted \\
E & 3.10 & $*$ & $*$ & 2.45 & $*$ & data \\
\hline Code & $\mathbf{Z 1}$ & $\mathbf{Z 2}$ & $\mathbf{Z 3}$ & $\mathbf{Z 4}$ & $\mathbf{Z 5}$ & $\mathbf{Z 6}$ \\
\hline A & 0.35 & 0.28 & 0.23 & 0.36 & 0.38 & 0.29 \\
B & 0.32 & 0.32 & 0.41 & 0.47 & 0.36 & 0.42 \\
C & 0.37 & 0.42 & 0.49 & 0.53 & 0.55 & 0.54 \\
D & 0.42 & 0.51 & 0.64 & 0.62 & 0.58 & 0.63 \\
E & 0.41 & 0.45 & 0.68 & 0.71 & 0.65 & 0.66 \\
F & 0.55 & 0.64 & 0.73 & 0.79 & 0.75 & 0.65 \\
\hline
\end{tabular}

Subsequent paragraphs must be indented by 0.64 ". Please do not double space, but use Layout tool tab to adjust the paragraph spacing and indentation. To evaluate the prediction of the NSCP formulas with the experiment results, the ratio of Pext against Pcalc were computed. This will be referred to as the strength ratio. The results are tabulated in Table 5. It can be seen that the values are greater than 1 for the $\mathrm{C}$-section. This means that the predictions using the NSCP formulas for the $\mathrm{C}$-sections are very conservative. However, for the Z-section CFS, the ratio Pexpt/Pcalc is lower than 1. This means that it would be an over-estimation to use the NSCP formulas for Z-section. Moreover, the average ratio for the $\mathrm{C}$-section is 2.56 while the average strength ratio for Z-section is 0.50 .

Displayed in Table 6 are the failure modes for each specimen classification. It is divided into two, the failure mode in the experiment and the failure mode in the computation. None of the specimens failed in yielding, hence, failures were only distortional buckling (DB) and torsionalflexural buckling (TF). Although DB is a form of local buckling, no other local buckling modes were observed. 
Table 6. Experimental Failure Mode and Calculated Failure Mode

\begin{tabular}{|c|c|c|c|c|c|c|c|c|c|c|c|c|}
\hline & $\begin{array}{r}\text { C1 } \\
\text { Exp }\end{array}$ & $\begin{array}{l}\text { C1 } \\
\text { Cal }\end{array}$ & $\begin{array}{r}\text { C2 } \\
\text { Exp }\end{array}$ & $\begin{array}{l}\text { C2 } \\
\text { Cal }\end{array}$ & $\begin{array}{r}\text { C3 } \\
\text { Exp }\end{array}$ & $\begin{array}{l}\text { C3 } \\
\text { Cal }\end{array}$ & $\begin{array}{r}\text { C4 } \\
\text { Exp }\end{array}$ & $\begin{array}{l}\mathrm{C4} \\
\text { Cal }\end{array}$ & $\begin{array}{l}\text { C5 } \\
\text { Exp }\end{array}$ & $\begin{array}{l}\text { C5 } \\
\text { Cal }\end{array}$ & & \\
\hline A & DB & DB & DB & $\mathrm{TF}$ & DB & $\mathrm{TF}$ & * & $\mathrm{TF}$ & $\mathrm{TF}$ & $\mathrm{TF}$ & \multicolumn{2}{|c|}{ Legend: } \\
\hline B & DB & DB & DB & $\mathrm{TF}$ & $\mathrm{TF}$ & $\mathrm{TF}$ & $\mathrm{TF}$ & $\mathrm{TF}$ & $\mathrm{TF}$ & $\mathrm{TF}$ & \multicolumn{2}{|c|}{$*$ Lost } \\
\hline $\mathrm{C}$ & DB & DB & DB & $\mathrm{TF}$ & $\mathrm{TF}$ & $\mathrm{TF}$ & $\mathrm{TF}$ & $\mathrm{TF}$ & TF & $\mathrm{TF}$ & \multicolumn{2}{|c|}{ data or } \\
\hline D & DB & $\mathrm{TF}$ & $\mathrm{TF}$ & $\mathrm{TF}$ & TF & $\mathrm{TF}$ & $*$ & $\mathrm{TF}$ & $\mathrm{TF}$ & $\mathrm{TF}$ & \multirow{2}{*}{\multicolumn{2}{|c|}{$\begin{array}{c}\text { corrupted } \\
\text { data }\end{array}$}} \\
\hline E & DB & TF & $*$ & $\mathrm{TF}$ & $*$ & $\mathrm{TF}$ & $\mathrm{TF}$ & $\mathrm{TF}$ & * & TF & & \\
\hline & $\mathrm{Z1}$ & $\mathrm{Z1}$ & $\mathbf{Z 2}$ & $\mathrm{Z2}$ & $\mathbf{Z 3}$ & $\mathbf{Z 3}$ & $\mathbf{Z 4}$ & $\mathbf{Z 4}$ & $\mathbf{Z 5}$ & $\mathbf{Z 5}$ & Z6 & Z6 \\
\hline & Exp & Cal & Exp & Cal & Exp & Cal & $\operatorname{Exp}$ & Cal & Exp & Cal & Exp & Cal \\
\hline A & $\mathrm{TF}$ & TF & $\mathrm{TF}$ & TF & $\mathrm{TF}$ & $\mathrm{TF}$ & DB & $\mathrm{TF}$ & $\mathrm{TF}$ & TF & $\mathrm{TF}$ & TF \\
\hline B & DB & $\mathrm{TF}$ & $\mathrm{TF}$ & $\mathrm{TF}$ & $\mathrm{TF}$ & $\mathrm{TF}$ & TF & $\mathrm{TF}$ & TF & $\mathrm{TF}$ & TF & TF \\
\hline C & $\mathrm{TF}$ & $\mathrm{TF}$ & $\mathrm{TF}$ & $\mathrm{TF}$ & $\mathrm{TF}$ & $\mathrm{TF}$ & DB & $\mathrm{TF}$ & TF & $\mathrm{TF}$ & $\mathrm{TF}$ & TF \\
\hline D & $\mathrm{TF}$ & $\mathrm{TF}$ & $\mathrm{TF}$ & $\mathrm{TF}$ & TF & $\mathrm{TF}$ & $\mathrm{TF}$ & $\mathrm{TF}$ & DB & $\mathrm{TF}$ & TF & $\mathrm{TF}$ \\
\hline B & $\mathrm{TF}$ & $\mathrm{TF}$ & $\mathrm{TF}$ & $\mathrm{TF}$ & TF & $\mathrm{TF}$ & DB & $\mathrm{TF}$ & $\mathrm{TF}$ & $\mathrm{TF}$ & $\mathrm{TF}$ & $\mathrm{TF}$ \\
\hline $\mathrm{F}$ & $\mathrm{TF}$ & TF & DB & $\mathrm{TF}$ & DB & TF & $\mathrm{TF}$ & $\mathrm{TF}$ & $\mathrm{TF}$ & $\mathrm{TF}$ & TF & TF \\
\hline
\end{tabular}

A comparison between the modes of failures was done to check for similarities between the computation and the experimental failure modes.

For C-section, the consistency of failures predicted by the computations and the actual failure in experiment is around $70 \%$. Furthermore, $45 \%$ of the population exhibited distortional buckling failure while 55\% exhibited torsional-flexural buckling.

For the Z-section, it was calculated that $81 \%$ of the population have similar experimental and calculated mode of failure. Only $19 \%$ of the total specimens exhibited distortional buckling failure while $81 \%$ exhibited torsional-flexural buckling.

Generally, DB happened for shorter lengths and TF occurred for longer lengths. However, this was not consistently observed in the experiment. It is observed that the experimental failure modes were not all predicted correctly by the computational method specified in the NSCP. However, all specimens that were calculated to fail in DB were predicted correctly by experiment. But, TF in the experiment was not consistently predicted.

\section{Evaluation of Strength through FEM}

In the previous section, it was found out that the strength of the $\mathrm{C}$-section was conservative estimated by the NSCP formulas. However, the opposite is observed for the Z-section. Hence to provide verification of which strength is more accurate whether experimental or computational, FEM analyses were conducted for the Z-section.

"FEM strengths" is the term used for the strength of the members that were obtained using the FEM analysis. This is denoted as Pfem. There were three general steps in determining the strength of a member. These are generation of the model and setting up of the boundary conditions, solving the model and gathering and analyzing the results. This is based on the work of MacDonald and Kulatunga [10]. Prior to analysis, an input for the modulus of elasticity, the yield strength, and density of $8027.3 \mathrm{~kg} / \mathrm{m}^{3}$ were inputted in the engineering data. The FEM results are summarized in Table 7. 
Table 7. FEM Strength Results (kN)

\begin{tabular}{ccccccc} 
Code & $\mathbf{Z 1}$ & $\mathbf{Z 2}$ & $\mathbf{Z 3}$ & $\mathbf{Z 4}$ & $\mathbf{Z 5}$ & $\mathbf{Z 6}$ \\
\hline A & 0.63 & 0.78 & 0.78 & 0.76 & 0.82 & 0.71 \\
B & 1.54 & 1.58 & 1.25 & 1.47 & 1.58 & 1.58 \\
C & 2.12 & 2.34 & 2.38 & 2.55 & 2.50 & 2.42 \\
D & 3.88 & 4.03 & 3.74 & 3.89 & 3.64 & 3.76 \\
E & 4.57 & 4.93 & 4.51 & 4.83 & 4.57 & 4.41 \\
F & 7.89 & 8.30 & 7.37 & 7.52 & 7.08 & 6.09 \\
\hline
\end{tabular}

The FEM results were then compared to the experimental results, and this is tabulated in Table 8. The experimental against computational strength ratio yielded an average of 0.50 while FEM against computational strength ratio resulted to 0.54. The experimental and FEM agreed well with an average strength ratio of 1.10. Hence it maybe said that the FEM gave better prediction as compared to the predictions using the NSCP formulas. Also, since the strength ratio is generally greater than 1.0, the FEM prediction is conservative.

\begin{tabular}{ccccccc}
\multicolumn{6}{c}{ Table 8. Comparison between FEM and Experimental Strength Results (Pexpt/Pfem) } \\
Code & $\mathbf{Z 1}$ & $\mathbf{Z 2}$ & $\mathbf{Z 3}$ & $\mathbf{Z 4}$ & $\mathbf{Z 5}$ & $\mathbf{Z 6}$ \\
\hline A & 2.60 & 1.76 & 1.19 & 1.57 & 1.46 & 1.11 \\
B & 1.56 & 1.30 & 1.55 & 1.27 & 0.88 & 0.92 \\
C & 1.51 & 1.25 & 1.15 & 0.98 & 0.98 & 0.88 \\
D & 1.28 & 1.07 & 1.11 & 0.85 & 0.78 & 0.74 \\
E & 1.11 & 0.84 & 1.06 & 0.84 & 0.74 & 0.70 \\
F & 1.03 & 0.83 & 0.79 & 0.69 & 0.66 & 0.61 \\
\hline
\end{tabular}

Using ANSYS, the member was modelled for analysis and boundary conditions were applied. Only half of the member was considered in the analysis to be able to generate similar conditions in the actual experiment. A remote displacement support B, was placed on one end which inhibited rotation and only allowed for horizontal movement (X \& Y). A displacement support, A, was also placed on the opposite end to inhibited horizontal movement (X \& Y) and allow all rotations in the $\mathrm{X}, \mathrm{Y} \& \mathrm{Z}$ directions including vertical movement. This configuration exhibits a pinned-pinned condition for the member. A force, B, was placed at the displacement support end in the form of a unit load. This set-up is illustrated in Figure 5a. To attain a more reliable result, a mesh size of $20 \mathrm{~mm}$ was used. A sample output of the FEM analysis is shown in the Figure 5b. The strength was analyzed using the Eigenvalue buckling analysis of ANSYS [10].

\section{Analysis of Results of Strength Evaluation}

The strength results were evaluated by comparing the experimental strength to computational strength. Shown in Figure 6 are plots of these comparisons so that the general trend can be established. The diagonal line in the graph is the equality line that represents the points at which the computational strength is equal to the experimental strength. Data points found below this equality line indicate over-estimate of the computational strength, and data points found above indicate under-estimate of the computational strength. Simply put, data points above are conservative or safe prediction of strengths, while those found below are not conservative or overestimation of the strengths. 


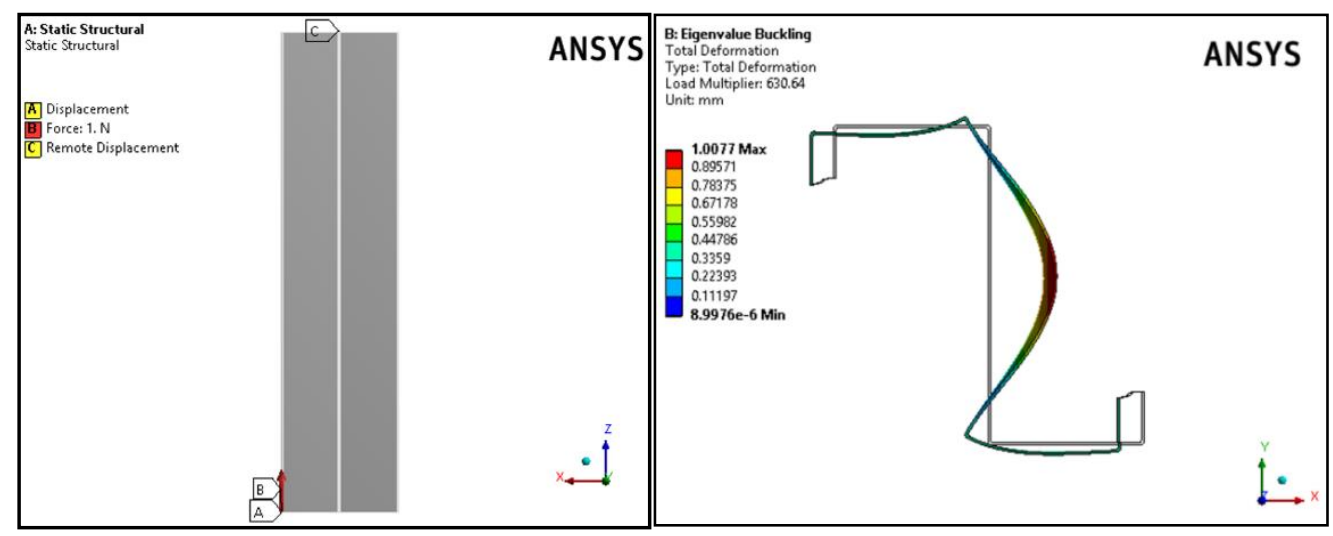

(a)

(b)

Figure 5. Sample run of FEM analysis showing deformation of the cross-section
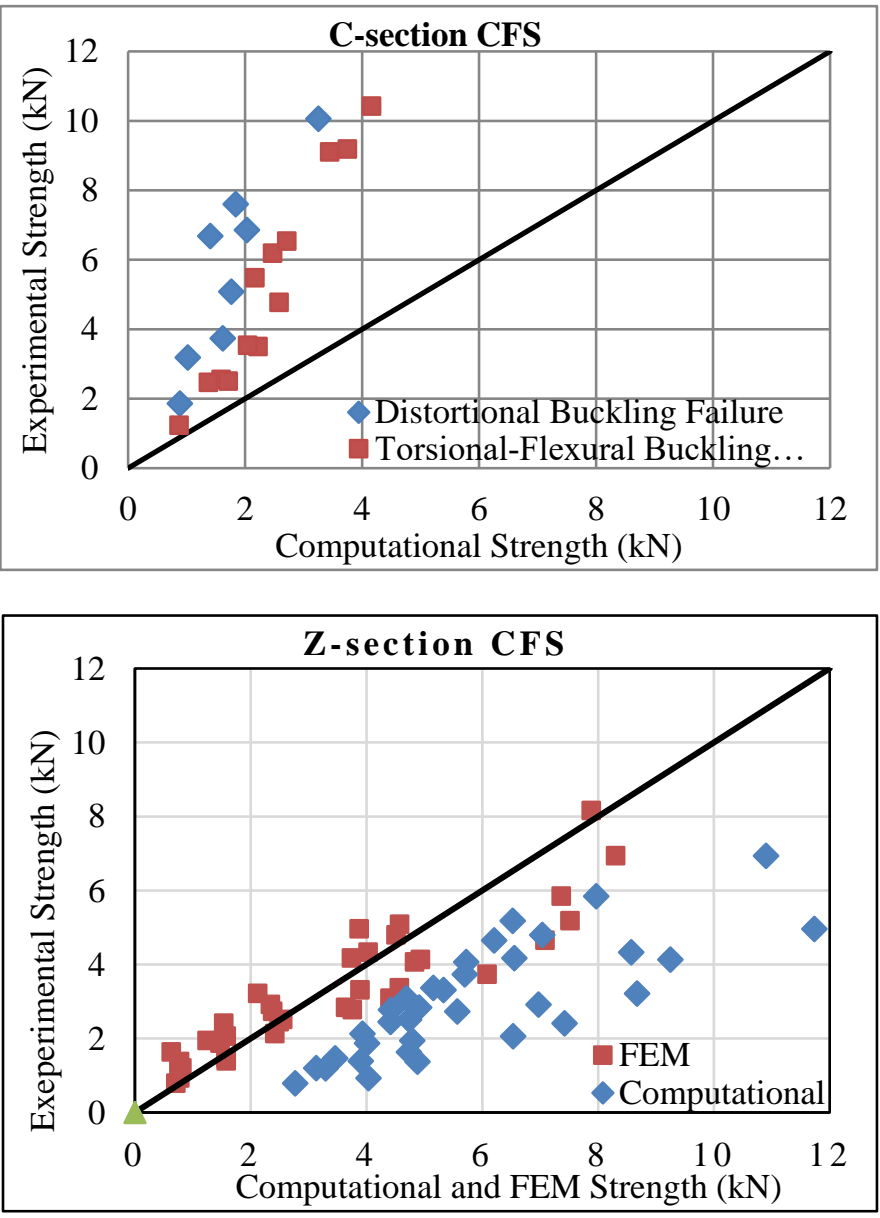

Figure 6. Comparison of strengths of c-section (top) and z-section (bottom)

The comparison of the compressive strength values for the C-sections displayed very conservative and low predictions as compared to the actual (experimental) compressive strength values. Distortional buckling failures have in general very low predictions resulting to having an actual strength of almost five times the predicted value. The nearest prediction for the actual 
strength is more than twice the predicted value. Sample groups with torsional-flexural buckling failures had nearer predictions. Torsional-flexural buckling computations are more accurate than distortional buckling failure predictions. Since the prediction of strength of the C-section CFS members is conservative, then the strength designs using the current NSCP provisions can be safely used.

For the Z-section the comparison of strength indicates that the NSCP formulas overestimate the strength. All data points are found under the equality line, meaning the computational or predicted strengths are higher than the experimental or actual strengths. The FEM/experimental strength was also plotted and showed good agreement between FEM and experimental strengths. Statistical analysis using ANOVA was conducted. The results indicate that the FEM and experimental strength had no significant difference while the computational strength had significant difference from both the experimental and FEM strength. A linear regression was done to show the slope for the best-fit line. The result shows a relatively linear relationship between the computational and experimental strength.

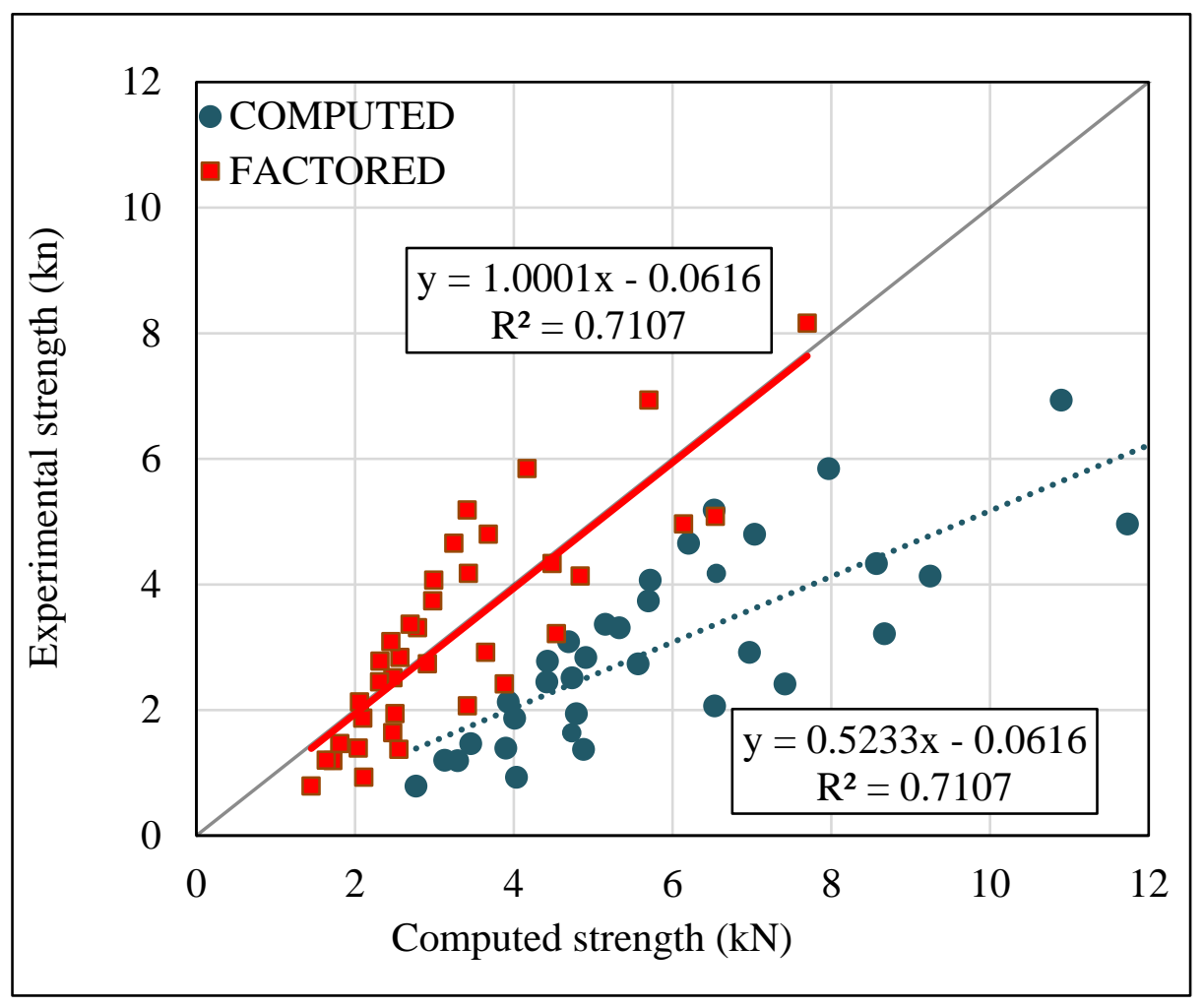

Figure 7. Strength results with factor $=0.52$ applied

In Figure 7, the plot of the computed strength against the experimental strength of Zsection is again plotted. This is represented by circular markers. The regression line (dotted line) shows a slope of 0.52. Thus, the provisions found in the NSCP for cold-formed steel members do not produce accurate strength predictions of the actual strength. The provisions must be modified and adjusted to predict the compressive strength of the cold-formed steel members manufactured in the Philippines. Hence, to be able to use the formulas stipulated in the Code that would lead to 
a safe design, a factor $=0.52$ can be applied to the computational strength. When this factor is applied, the computational strength would result to a "factored strength" that agrees well with experimental strength as illustrated in Figure 7. The solid orange line is the regression line after applying the factor to the original computational strength. The dotted line is the original computational strength.

\section{Conclusions}

The local-global interaction of the buckling failure modes was dependent on the thickness and length of the member. The strength increases as the thickness increases. Consequently, it was found that the longer length contributed to the occurrence of torsional flexural buckling, while the thinner CFS tends to contribute more to the occurrence of distortional buckling. Moreover, smaller lengths exhibit higher strengths.

Although not consistent, the comparison of failure modes shows relatively good agreement, $70 \%$ for the $\mathrm{C}$-section and $81 \%$ for the $\mathrm{Z}$-section. It was also observed that dominant failure mode was torsional-flexural buckling.

A comparison between the three strength evaluation methods (experimental, computational, and FEM) is done on the averages of the specimens' strengths. The predictions made using the NSCP formulas were too low for C-section CFS and too high for Z-section CFS. Thus, it may be concluded that the use of the NSCP formula (in its present form) may be over conservative for the $\mathrm{C}$-section and non-conservative for the $\mathrm{Z}$-section. As a side note, the average ratio between the experimental and FEM results was 1.10 indicating good agreement and verifying the experimental results.

Lastly, since the code-based prediction for the C-section is over-conservative, it may be concluded that it can be used in its present form. However, for the Z-section, a modification factor of 0.52 may be used by multiplying it to strength obtained based on the NSCP to achieve a relatively conservative result. This may be done to allow for the use of the provisions of the NSCP without changing the stipulated equations.

\section{Acknowledgment}

The authors would like to acknowledge the faculty and staff of the Civil Engineering Department, Gokongwei College of Engineering, De La Salle University, Manila. Special thanks also to ERDT for providing research grants. The authors also acknowledge those who helped in the research process, especially in doing the experimental tests.

\section{References}

[1] J. Davies, "Recent research advances in cold-formed steel structures," Journal of Constructional Steel Research, Vol. 55, No. 1-3, pp. 267-288, 2000. doi: 10.1016/S0143974X(99)00089-9

[2] J. Rondal, "Cold-formed steel members and structures: General report," Journal of Constructional Steel Research, Vol. 55, pp. 155-158, 2000. doi: 10.1016/S0143974X(99)00083-8 
[3] D. Dubina, V. Ungureanu, and R. Landolfo, Design of Cold-Formed Steel Structures, $1^{\text {st }}$ Edition, Berlin: European Convention for Constructional Steelwork, Mem Martins, Portugal, 2012.

[4] G.J. Hancock, "Cold-formed steel structures," Journal of Constructional Steel Research, Vol. 59, pp. 473-487, 2003. doi: 10.1016/S0143-974X(02)00103-7

[5] Association of Structural Engineers of the Philippines, "Chapter 5: Structural Steel," In National Structural Code of the Philippines, Volume 1, $7^{\text {th }}$ Edition, Association of Structural Engineers of the Philippines (ASEP), pp. 228-326, 2015.

[6] ASTM International, Standard Test Methods for Tension Testing of Metallic Materials (ASTM E8 / E8M-16a), West Conshohocken, Pennsylvania, United States, 2016. doi: 10.1520/E0008_E0008M-16A

[7] J.M. De Jesus, and B. Lejano, "An investigation on the strength of axially loaded coldformed steel Z-Sections," International Journal of GEOMATE, Vol. 14, No. 42, pp. 30-36, 2018.

[8] A.P. Yu, and B. Lejano, "Investigation on the strength of cold-formed steel C-section in compression," In: International Conference on Advances on Civil, Structural, Environmental and Bio-Technology CSEB 2014, Kuala Lumpur, Malaysia, pp. 137-141, 2014.

[9] E. Batista, "Local-global buckling interaction procedures for the design of cold-formed columns: Effective width and direct method integrated approach," Thin-Walled Structures, Vol. 47, pp. 1218-1231, 2009. doi: 10.1016/j.tws.2009.04.004

[10] M. MacDonald, and M. Kulatunga, "Finite element analysis of cold-formed steel structural members with performations subjected to compression loading," Mechanics and Mechanical Engineering, Vol. 17, No. 2, pp. 127-139, 2013 [Online]. Available: http://www.kdm.p.lodz.pl/articles/2013/17_2_2M_K.pdf

\section{Appendix}

According to the National Structural Code of the Philippines, the axial strength of concentrically loaded compression members is the minimum value between Equation (1), (2), (3) or (4). The nominal strength depends on the axial strength that governs. Thus, this determines the buckling mode of failure that is predicted to occur in the member.

Yielding Failure

$$
P_{n}=A_{e} F_{y}
$$

Where, $\quad A_{e}=$ effective area

$F_{y}=$ yield stress of the steel

\section{Torsional or Flexural-Torsional Buckling}

$$
P_{n}=A_{e} F_{n}
$$

Where, $\quad F_{n}=$ reduced stress 
For $\lambda_{c} \leq 1.5$,

$$
F_{n}=\left(0.658 \lambda_{c}^{2}\right) F_{y}
$$

For $\lambda_{c}>1.5$,

$$
F_{n}=\left(\frac{0.877}{\lambda_{c}^{2}}\right) F_{y}
$$

Where, $\quad \lambda_{c}=$ slenderness ratio $=\sqrt{\frac{F_{y}}{F_{e}}}$

The elastic critical buckling stress is computed as follows:

$$
F_{e}=\text { smaller of }\left\{\begin{array}{c}
\sigma_{t}=\frac{1}{A r_{o}{ }^{2}}\left[G J+\frac{\pi^{2} E C_{w}}{\left(k_{t} L_{t}\right)^{2}}\right](\text { Section 553.3.1.2.1) } \\
F_{e}=\frac{\pi^{2} E}{\left(\frac{K L}{r}\right)^{2}}(\text { Section 553.4.1.1) }
\end{array}\right\}
$$

Where, $\quad r=$ radius of gyration of unreduced area

$r_{x}, r_{y}=$ radius of gyration about $\mathrm{x}$ and $\mathrm{y}$-axis respectively $=\sqrt{\frac{I_{x}}{A}}, \sqrt{\frac{I_{y}}{A}}$

$r_{o}=$ polar radius of gyration about shear center

$=\sqrt{r_{x}^{2}+r_{y}^{2}+x_{o}^{2}}$

$x_{o}=$ distance from shear center to centroid of section $=0$

$A=$ full unreduced area of section

$G=$ Shear modulus

$J=$ Torsional Constant $=\frac{t^{3}}{3}\left(2 b_{o}+2 D+h_{o}\right)$

$C_{w}=$ Torsional warping constant $=\frac{b_{o}{ }^{2} t}{12\left(2 b_{o}+h_{o}+2 D\right)}\left[h_{o}{ }^{2}\left(b_{o}{ }^{2}+\right.\right.$

$\left.2 b_{o} h_{o}+4 b_{o} D+6 h_{o} D\right)+4 D^{2}\left(3 b_{o} h_{o}+3 h_{o}{ }^{2}+4 b_{o} D+\right.$

$\left.\left.2 h_{o} D+D^{2}\right)\right]$ (Dubina et al, 2012)

$k_{t}=$ effective length factor from twisting

$L_{t}=$ unbraced length from twisting

$K=$ effective length factor

\section{Distortional Buckling}

$$
\begin{array}{ll}
\text { For } \lambda_{d} \leq 0.561, & P_{n}=P_{y} \\
\text { For } \lambda_{d}>0.561, & P_{n}=\left[1-0.25\left(\frac{P_{c r d}}{P_{y}}\right)^{0.6}\right]\left(\frac{P_{c r d}}{P_{y}}\right)^{0.6} P_{y}
\end{array}
$$

$$
\text { Where, } \quad \begin{aligned}
\lambda_{d} & =\sqrt{\frac{P_{y}}{P_{c r d}}} \\
P_{y} & =\text { nominal strength due to yielding }
\end{aligned}
$$




$$
\begin{aligned}
& P_{c r d}=A_{g} F_{d} \\
& A_{g}=\text { full unreduced area of section } \\
& F_{d}=\text { Elastic distortional buckling stress } \\
& =k_{d}\left[\frac{\pi^{2} E}{12\left(1-\mu^{2}\right)}\right]\left(\frac{t}{b_{o}}\right)^{2} \\
& k_{d}=0.05 \leq\left(0.01 \frac{b_{o} D \sin \theta}{h_{o} t}\right)^{1.4} \leq 8.0 \\
& \theta=\text { angle of rotation of the lip stiffener } \\
& h_{o}, b_{o}=\text { Out-to-out web depth and flange width }
\end{aligned}
$$

